\title{
Closure Property of Probabilistic Turing Machines and Alternating Turing Machines with Subalgorithmic Spaces
}

\author{
by \\ Géza Horváth \\ Katsushi Inoue \\ Akira Ito \\ and \\ Yue Wang
Department of Computer Science and Systems Engineering Faculty of Engineering Yamaguchi University \\ Ube, 755-8611 Japan
}

\author{
Mailing Address: \\ Katsushi Inoue \\ Department of Computer Science and Systems Engineering \\ Faculty of Engineering \\ Yamaguchi University \\ Ube, 755-8611 Japan \\ Email: inoue@csse.yamaguchi-u.ac.jp
}




\section{Introduction}

Freivalds [4] showed a surprising result of the language $\left\{a^{n} b^{n} \mid n \geq 1\right\}$ being recognized by a two-way Monte Carlo finite automaton (i.e., a two-way probabilistic finite automaton with error probability less than $1 / 2$ ). This result influenced many subsequent papers $[3,8,14]$. As far as we know, it is unknown whether the classes of languages recognized by $o(\log n)$ space bounded two-way Monte Carlo Turing machines [5] and two-way probabilistic Turing machines [7] are closed under concatenation, Kleene closure, and length-preserving homomorphism. By using an adaptation of the proof of the above result, and a separation result by Frievald and Karpinski [5], Section 3 of this paper shows that (1) the class of languages recognized by $o(\log n)$ space-bounded two-way Monte-Carlo Turing machines is not closed under these operations, and (2) the class of languages recognized by $o(\log \log n)$ space-bounded two-way unbounded error probabilistic Turing machines is not closed under these operations.

Many investigations of alternating Turing machines (aTm's) with subalgorithmic spaces have been made $[1,2,6,10,12,13]$. Chang, Ibarra and Ravikumar [2] showed that the language $\left\{0^{n} 10^{n} \mid n \geq 1\right\}$ can be accepted by a weakly log log $n$ space-bounded one-way aTm. Ito, Inoue, and Takanami [10] showed that there exists a language accepted by a strongly $\log \log n$ space-bounded two-way aTm, but not accepted by any weakly $o(\log n)$ space-bounded one-way aTm. Iwama [12] showed that the languages accepted by weakly $o(\log \log n)$ space-bounded two-way aTm's are regular. Furthermore, Braunmühl, Genger and Rettinger [1], Geffert [6], and Liśkiewicz and Reischuk [13] showed that the alternation hierarchy for aTm's with space bounds between $\log \log n$ and $\log n$ is infinite.

Section 4 of this paper answers an open question [10] of whether the class of languages accepted by $\mathrm{S}(n)$ space-bounded two-way aTm's is closed under concatenation, Kleene closure, and length-preserving homomorphism for $\log \log n \leq$ $\mathrm{S}(n) \leq o(\log n)$, and shows that the class mentioned above is not closed under these operations.

\section{Preliminaries}

For each word $w,|w|$ denotes the length of $w$, and for each set $\mathrm{T},|\mathrm{T}|$ denotes the number of elements of T. See [9] for undefined terms.

A two-way probabilistic Turing machine we consider here has a read-only input tape delimited by the left endmarker $\phi$ and the right endmaker $\$$, and a semi-infinite read-write work tape, initially blank. Of course, the input head of the machine can move left or right. See [7] for the definitions of this machine. As in Freivalds and Karpinski [5], we distinguish between two types of two-way probabilistic Turing machines: Two-way Monte Carlo Turing machines and two-way unbounded error probabilistic Turing machines.

We say that a two-way Monte Carlo Turing machine $\mathrm{M}$ recognizes language $\mathrm{L}$ in space $\mathrm{S}(n)$ if there is a positive constant $\varepsilon$ such that: 
(1) for any $x \in \mathrm{L}$, the probability of the event "M accepts $x$ in space not exceeding $\mathrm{S}(|x|)$ " exceeds $\frac{1}{2}+\varepsilon$, and

(2) for any $x \notin \mathrm{L}$, the probability of the event "M rejects $x$ in space not exceeding $\mathrm{S}(|x|)$ " exceeds $\frac{1}{2}+\varepsilon$.

We say that a two-way unbounded error probabilistic Turing machine $\mathrm{M}$ recognizes language $\mathrm{L}$ in space $\mathrm{S}(n)$ if:

(1) for any $x \in \mathrm{L}$, the probability of the event "M accepts $x$ in space not exceeding $\mathrm{S}(|x|)$ " exceeds $\frac{1}{2}$, and

(2) for any $x \notin \mathrm{L}$, the probability of the event "M rejects $x$ in space not exceeding $\mathrm{S}(|x|)$ " exceeds $\frac{1}{2}$.

Let MSPACE $(\mathrm{S}(n))$ (resp., PSPACE $(\mathrm{S}(n))$ ) denote the class of languages recognized by two-way Monte Carlo Turing machines (resp., two-way unbounder error probabilistic Turing machines) in space $\mathrm{S}(n)$.

A two-way alternating Turing machine (2aTm) we consider here has a readonly input tape delimited by the left endmaker $\not{C}$ and the right endmaker $\$$, an input head which can move left or right on the input tape, and a semi-infinite read-wrie work tape, initially blank. See $[1,2,6,10,12,13]$ for the definition of 2aTm's.

We can view the computation of a $2 \mathrm{aTm} \mathrm{M}$ as a tree whose nodes are labeled by configurations. A configuration of $\mathrm{M}$ is of the form $(i,(q, \gamma, k))$, where $i$ is the input tape head position, and component $(q, \gamma, k)$ represents the state of the finite control, the non-blank contents of the work tape, and the work tape head position. If $q$ is the state associated with configuration $c$, then $c$ is said to be a universal (resp., existential, accepting) configuration if $q$ is universal (resp., existential, accepting) state. The initial configuration of $\mathrm{M}$ is $I_{M}=\left(0,\left(q_{0}, \lambda, 1\right)\right)$, where $q_{0}$ is the initial state of $\mathrm{M}$ and $\lambda$ is the null string. A computation tree of $\mathrm{M}$ on input $w$ is a tree such that the root is labeled by $I_{M}$ and the children of any nonleaf node labeled by a universal (resp., existential) configuration include all (resp., one) of the immediate successors (of $\mathrm{M}$ on $w$ ) of that configuration. A computation tree is accepting if it is finite and all the leaves are labeled by accepting configurations. $\mathrm{M}$ accepts an input $w$ if there is an accepting computation tree of $\mathrm{M}$ on $w$.

Let $l$ be a non-negative integer and $c=(i,(q, \gamma, k))$ be a configuration of M. $c$ is $l$ space-bounded if $|\gamma| \leq l$.

A computation tree of $\mathrm{M}$ (on some input) is $l$ space-bounded if each node of the tree is labeled by a $l$ space-bunded configuration of M.

Let $\mathrm{S}(n): N \rightarrow N \cup\{0\}$ be a function, where $N$ denotes the set of all the positive integers. M is weakly $S(n)$ space-bounded if for every input $w$ of length $n, n \geq 1$, that is accepted by $\mathrm{M}$, there exists an $\mathrm{S}(n)$ space-bounded accepting computation tree of $\mathrm{M}$ on $w$. M is strongly $S(n)$ space-bounded if for every input $w$ of length $n$ (accepted by $\mathrm{M}$ or not), $n \geq 1$, any computation tree of $\mathrm{M}$ on $w$ is $\mathrm{S}(n)$ space-bounded.

Let weak-ASPACE $(\mathrm{S}(n))$ (resp., strong-ASPACE$(\mathrm{S}(n))$ ) denote the class of languages accepted by weakly(resp., strongly) S(n) space-bounded 2aTm's. 


\section{Closure Property of Probabilistic Turing Machines}

This section shows that MSPACE $(o(\log n))$ and PSPACE $(o(\log \log n))$ are not closed under concatenation, Kleene closure, and length preserving homomorphism. The following two lemmas (which were given by Freivalds and Karpinski [5]) are used to get our desired result.

Lemma 3.1. Let $\mathrm{A}, \mathrm{B} \subseteq \Sigma^{*}$ with $\mathrm{A} \cap \mathrm{B}=\emptyset$ (empty set). Suppose that there are an infinite set I of positive integers, and functions $\mathrm{G}(n), \mathrm{H}(n)$ such that $\mathrm{G}(n)$ is a fixed polynomial in $n$, and for each $n \in \mathrm{I}$, there is a set $\mathrm{W}(n)$ of words in $\Sigma^{*}$ such that:

(1) $|w| \leq \mathrm{G}(n)$ for each word $w \in \mathrm{W}(n)$,

(2) there is a constant $c>1$ such that $|\mathrm{W}(n)| \geq c^{n}$ for each $n \in \mathrm{I}$, and

(3) for every $n \in \mathrm{I}$ and every $w, w^{\prime} \in \mathrm{W}(n)$ with $w \neq w^{\prime}$, there are words $u, v \in \Sigma^{*}$ such that:

(a) $|u w v| \leq \mathrm{H}(n),\left|u w^{\prime} v\right| \leq \mathrm{H}(n)$, and

(b) either $\left\{\begin{array}{l}u w v \in \mathrm{A} \\ u w^{\prime} v \in \mathrm{B}\end{array}\right.$ or $\left\{\begin{array}{l}u w v \in \mathrm{B} \\ u w^{\prime} v \in \mathrm{A} .\end{array}\right.$

Then, if a two-way Monte Carlo Turing machine with space bound $\mathrm{S}(n)$ separates $\mathrm{A}$ and $\mathrm{B}$, then $\mathrm{S}(\mathrm{H}(n))$ cannot be $o(\log n)$.

Lemma 3.2. Let $A, B \subseteq \Sigma^{*}$ with $A \cap B=\emptyset$. Suppose that there is an infinite set I of positive integers and a function $\mathrm{H}(n)$ such that for each $n \in \mathrm{I}$, there is an ordered set of pairs of words $\mathrm{W}(n)=\left\{\left(u_{1}, v_{1}\right),\left(u_{2}, v_{2}\right), \ldots,\left(u_{n}, v_{n}\right)\right\}$ such that for every string $\gamma(1) \gamma(2) \ldots \gamma(n) \in\{0,1\}^{n}$, there is a word $w$ such that

$$
\left\{\begin{array}{l}
u_{i} w v_{i} \in \mathrm{A}, \text { if } \gamma(i)=1 \\
u_{i} w v_{i} \in \mathrm{B}, \text { if } \gamma(i)=0
\end{array}\right.
$$

and $\left|u_{i} w v_{i}\right| \leq \mathrm{H}(n)$ for all $i \in\{1,2, \ldots, n\}$. Then, if a two-way unbounded error probabilistic Turing machine with space bound $\mathrm{S}(n)$ separates $\mathrm{A}$ and $\mathrm{B}$, then $\mathrm{S}(\mathrm{H}(n))$ cannot be $o(\log \log n)$.

The following lemma is a key one.

\section{Lemma 3.3. Let}

$\mathrm{L}_{1}=\left\{a^{m_{1}} 1 a^{m_{2}} 1 \ldots 1 a^{m_{k}} \mid k \geq 2 \& \forall i(1 \leq i \leq k)\left[m_{i} \geq 1\right] \& m_{1}=m_{k}\right\}$,

$\mathrm{L}_{2}=\left\{1 a^{m} \mid m \geq 1\right\}^{*}$,

$\mathrm{L}_{3}=\left\{a^{m_{1}} 1 a^{m_{2}} 1 \ldots 1 a^{m_{k}} \mid k \geq 2 \& \forall i(1 \leq i \leq k)\left[m_{i} \geq 1\right]\right\}$, and

$\mathrm{L}_{4}=\left\{a^{m_{1}} b_{1} a^{m_{2}} b_{2} \ldots b_{k-1} a^{m_{k}} \mid k \geq 2 \& \forall i(1 \leq i \leq k)\left[m_{i} \geq 1\right] \&\right.$

$\left.\exists j(1 \leq j \leq k-1)\left[b_{j}=2 \& \forall r(1 \leq r \leq k-1, r \neq j)\left[b_{r}=1\right] \& m_{1}=m_{j+1}\right]\right\}$.

Then,

(1) $\mathrm{L}_{1} \in \operatorname{MSPACE}(0)$, and thus $\in \operatorname{PSPACE}(0)$,

(2) $\mathrm{L}_{1} \cup \mathrm{L}_{2} \in \operatorname{MSPACE}(0)$, and thus $\in \operatorname{PSPACE}(0)$,

(3) $\mathrm{L}_{3} \in \operatorname{MSPACE}(0)$, and thus $\in \operatorname{PSPACE}(0)$,

(4) $\mathrm{L}_{4} \in \operatorname{MSPACE}(0)$, and thus $\in \operatorname{PSPACE}(0)$,

(5) $\mathrm{L}_{1} \mathrm{~L}_{2} \notin \operatorname{MSPACE}(o(\log n))$, and

(6) $\mathrm{L}_{1} \mathrm{~L}_{2} \notin \operatorname{PSPACE}(o(\log \log n))$.

Proofs of (1),(2), and (4): By an adaptation of the proof of the fact [4] that $\left\{a^{n} b^{n} \mid n \geq 1\right\} \in \operatorname{MSPACE}(0)$. 
Proof of (3): Obvious.

Proof of (5): We first note that $\mathrm{L}_{1} \mathrm{~L}_{2}=\left\{a^{m_{1}} 1 a^{m_{2}} 1 \ldots 1 a^{m_{k}} \mid k \geq 1 \& \forall i(1 \leq\right.$ $\left.i \leq k)\left[m_{i} \geq 1\right] \& \exists j(2 \leq j \leq k)\left[m_{1}=m_{j}\right]\right\}$.

For any integer $n \geq 1$, let $\mathrm{V}(n)=\left\{1 a^{m_{1}} 1 a^{m_{2}} 1 \ldots 1 a^{m_{n}} \in\{1, a\}^{+} \mid \forall i(1 \leq\right.$ $\left.i \leq n)\left[1 \leq m_{i} \leq n\right]\right\}$. For each $w=1 a^{m_{1}} 1 a^{m_{2}} 1 \ldots a^{m_{n}} \in \mathrm{V}(n)$ let contents $(w)=$ $\left\{a^{j} \mid j=m_{i}\right.$ for some $\left.i(1 \leq i \leq n)\right\}$. Divide $\mathrm{V}(\mathrm{n})$ into contents-equivalence classes by making $w$ and $w^{\prime}$ contents-equivalent if contents $(w)=\operatorname{contents}\left(w^{\prime}\right)$. There are

$$
\text { contents }(n)=\left(\begin{array}{l}
n \\
1
\end{array}\right)+\left(\begin{array}{l}
n \\
2
\end{array}\right)+\ldots+\left(\begin{array}{l}
n \\
n
\end{array}\right)=2^{n}-1
$$

contents-equivalence classes of words in $\mathrm{V}(n)$. We denote by $\mathrm{W}(n)$ the set of all the representatives arbitrarily chosen from these contents $(n)$ contentsequivalence classes. For each word $w \in \mathrm{W}(n),|w| \leq \mathrm{G}(n) \triangleq(n+1) n$, which is a fixed polynomial in $\mathrm{n}$. Let $\mathrm{I}$ be the set of positive integers greater than or equal to 2 . Thus, for any $n \in \mathrm{I},|\mathrm{W}(n)|=\operatorname{contents}(n)=2^{n}-1 \geq c^{n}$ for some constant $c>1$. It is easily seen that for every $n \in \mathrm{I}$ and every $w, w^{\prime} \in \mathrm{W}(n)$ with $w \neq w^{\prime}$, there are words $u=a^{k}(1 \leq k \leq n), v=\epsilon$ such that

(a) $|u w v| \leq \mathrm{H}(n) \triangleq \mathrm{G}(n)+n,\left|u w^{\prime} v\right| \leq \mathrm{H}(n)$, and

(b) either $\left\{u w v \in \mathrm{L} \& u w^{\prime} v \in \overline{\mathrm{L}}\right\}$ or $\left\{u w^{\prime} v \in \mathrm{L} \& u w v \in \overline{\mathrm{L}}\right\}$,

where for any language $\mathrm{T}, \overline{\mathrm{T}}$ denotes the complement of $\mathrm{T}$.

Thus, by Lemma 3.1, if a two-way Monte Carlo Turing machine with space bound $\mathrm{S}(n)$ recognizes $\mathrm{L}_{1} \mathrm{~L}_{2}$, then $\mathrm{S}(\mathrm{H}(n))$ can not be $o(\log n)$, and thus $\mathrm{S}(n)$ can not be $o(\log n)$. This completes the proof of ' $\mathrm{L}_{1} \mathrm{~L}_{2} \notin \operatorname{MSPACE}(o(\log n))$ '.

Proof of (6): Let I be the set of positive integers, and let $\mathrm{H}$ : $\mathrm{I} \rightarrow \mathrm{I}$ be the function such that $\mathrm{H}(n)=2 n+\frac{n(n+1)}{2}$.

For each $n \in \mathrm{I}$, let $\overline{\mathrm{W}}(n)=\left\{\left(u_{1}, v_{1}\right),\left(u_{2}, v_{2}\right), \ldots,\left(u_{n}, v_{n}\right)\right\}$ be the ordered set of pairs of words such that for each $i, 1 \leq i \leq n, u_{i}=a^{i}$ and $v_{i}=\epsilon$.

Furthermore, for the string $\alpha(1) \alpha(2) \ldots \alpha(n) \in\{0,1\}^{n}$, let $0<k_{1}<k_{2}<\ldots<k_{l}$ be all the values of $i$ such that $\alpha(i)=1$, and $w_{\alpha(1) \alpha(2) \ldots \alpha(n)}=1 a^{k_{1}} 1 a^{k_{2}} \ldots 1 a^{k_{l}}$ be the string corresponding to $\alpha(1) \alpha(2) \ldots \alpha(n)$.

It is easy to see that for each $n \in \mathrm{I}$ and for each string $\alpha(1) \alpha(2) \ldots \alpha(n) \in\{0,1\}^{n}$,

$$
\left\{\begin{array}{l}
u_{i} w_{\alpha(1) \alpha(2) \ldots \alpha(n)} v_{i} \in \mathrm{L}_{1} \mathrm{~L}_{2} \text { if } \alpha(i)=1, \\
u_{i} w_{\alpha(1) \alpha(2) \ldots \alpha(n)} v_{i} \in \overline{\mathrm{L}_{1} \mathrm{~L}_{2}} \text { if } \alpha(i)=0,
\end{array}\right.
$$

and $\left|u_{i} w_{\alpha(1) \alpha(2) \ldots \alpha(n)} v_{i}\right| \leq \mathrm{H}(n)$ for all $i \in\{1,2, \ldots, n\}$.

Thus by Lemma 3.2, if a two-way unbounded error probabilistic Turing machine with space bound $\mathrm{S}(n)$ recognizes $\mathrm{L}_{1} \mathrm{~L}_{2}$, then $\mathrm{S}(\mathrm{H}(n))$ can not be $o(\log \log n)$ and thus $\mathrm{S}(n)$ can not be $o(\log \log n)$. This completes the proof of ' $\mathrm{L}_{1} \mathrm{~L}_{2} \notin \operatorname{PSPACE}(o(\log \log n))$ '.

By using Lemma 3.3., we can get the following theorem.

Theorem 3.1. $\operatorname{MSPACE}(o(\log n))$ and PSPACE$(o(\log \log n))$ are not closed under concatenation, Kleene closure, and length-preserving homomorphism. 
Proof. Let $\mathrm{L}_{i}, i \in\{1,2,3,4\}$, be the languages described in Lemma 3.3.

Concatenation: Nonclosure under concatenation follows from Lemma 3.3 (1), (5) and (6), and from the obvious fact that $\mathrm{L}_{2} \in \operatorname{MSPACE}(o(\log n)) \cap$

$\operatorname{PSPACE}(o(\log \log n))$.

Kleene closure: It follows that $\left(\mathrm{L}_{1} \cup \mathrm{L}_{2}\right)^{*} \cap \mathrm{L}_{3}=\mathrm{L}_{1} \mathrm{~L}_{2} \notin \operatorname{MSPACE}(o(\log n)) \cup$ $\operatorname{PSPACE}(o(\log \log n))$ (from Lemma $3.3(5)$ and (6)). From this, Lemma $3.3(2)$ and (3), and from the obvious fact that $\operatorname{MSPACE}(o(\log n))$ and PSPACE $(o(\log \log n))$ are closed under intersection with regular languages, nonclosure under Kleene closure follows.

Length-preserving homomorphism: Nonclosure under length-preserving homomorphism follows from Lemma $3.3(4),(5)$ and (6), and from the fact that $g\left(\mathrm{~L}_{4}\right)$ $=\mathrm{L}_{1} \mathrm{~L}_{2}$, where $g:\{1,2, a\} \rightarrow\{1, a\}$ is a length-preserving homomorphism such that $g(1)=g(2)=1$ and $g(a)=a$.

\section{Closure Property of ASPACE $(o(\log n))$}

This section shows that weak-ASPACE $(\mathrm{S}(n))$ and strong-ASPACE $(\mathrm{S}(n))$ are not closed under concatenation, Kleene closure, and length-preserving homomorphism for any $\log \log n \leq \mathrm{S}(n)=o(\log n)$. This result answers an open question in [10].

We first introduce a new idea of "rejecting computation tree" which was introduced in [11].

Given a $2 \mathrm{aTm} \mathrm{M}$, we write $c_{M, x} c^{\prime}$ if configuration $c^{\prime}$ is derived from configuration $c$ in one step of $\mathrm{M}$ on an input tape $x$. Let $\mathrm{C}_{M}$ be the set of all the configurations of M. For each $c \in \mathrm{C}_{M}$, let $\operatorname{Succ}_{M, x}(c)=\left\{c^{\prime} \in C_{M}|c|_{M}, c^{\prime}\right\}$. If $\operatorname{Succ}_{M, x}(c)=\emptyset$, then $c$ is said to be a halting configuration of M on $x$.

Let $l$ be a non-negative integer. An $l$ space-bounded rejecting computation tree of $\mathrm{M}$ on input $x$ is a (possibly infinite) nonempty labeled tree with the following properties:

(1) Each internal node $v$ (non leaf node) of the tree is labeled with an $l$ spacebounded configuration of $\mathrm{M}$, label $(v)$.

(2) The root node is labeled with $\mathrm{I}_{M}$.

(3) If $v$ is an internal node, and $\operatorname{label}(v)$ is universal, then $v$ has exactly one child $u$ such that $l a b e l(u) \in \operatorname{Succ}_{M, x}(\operatorname{label}(v))$.

(4) If $v$ is an internal node, $\operatorname{label}(v)$ is existential and $\operatorname{Succ}_{M, x}(\operatorname{label}(v))=$ $\left\{c_{1}, c_{2}, \ldots, c_{k}\right\}$, then $v$ has exactly $k$ children $v_{1}, v_{2}, \ldots, v_{k}$ such that $l a b e l\left(v_{i}\right)=$ $c_{i}(1 \leq i \leq k)$.

(5) Each leaf node is a halting configuration which is not accepting, or a configuration which is not $l$ space-bounded.

A reduced graph of $l$ space-bounded rejecting computation tree (abbreviated by $\mathrm{RG}(l))$ of $\mathrm{M}$ on input $x$ is a finite, labeled directed multi-graph $\mathrm{G}=\left(\mathrm{V}^{\prime}, \mathrm{E}\right.$, , label $\mid$ 
V') obtained from an $l$ space bounded rejecting computation tree $\mathrm{T}=(\mathrm{V}, \mathrm{E}$, label $)$ of $\mathrm{M}$ on $x$ by identifying nodes $v$ and $v^{\prime}$ such that label $(v)=\operatorname{label}\left(v^{\prime}\right)$ where $\mathrm{V}^{\prime} \subseteq \mathrm{V}$ and the labeling function label $\mid \mathrm{V}^{\prime}: \mathrm{V}^{\prime} \rightarrow \mathrm{C}_{M}$ is injective.

For any directed graph $G$, let $V(G)$ and $E(G)$ denote the sets of nodes and edges of $\mathrm{G}$, respectively.

Let $\delta_{G}^{-}=\left\{v^{\prime} \in V(G) \mid\left(v^{\prime}, v\right) \in E(G)\right\}$ for each node $v \in \mathrm{V}(\mathrm{G})$. Obviously, for a reduced graph of $l$ space-bounded rejecting computation tree, there exists at most one node $v$ such that $\delta_{G}^{-}(v)=0$ labeled with $\mathrm{I}_{M}$.

Let $\delta_{G}^{+}(v)=\left\{v^{\prime} \in V(G) \mid\left(v, v^{\prime}\right) \in E(G)\right\}$ for each node $v \in \mathrm{V}(\mathrm{G})$. An $\operatorname{RG}(l)$ $\mathrm{G}$ is regular if $\left|\delta_{G}^{+}(v)\right|=1$ for each node $v$ labeled with a universal configuration.

The following fact is used to get our desired result.

Fact 4.1. Let $\mathrm{M}$ be a $2 \mathrm{aTm}, x$ be a word, and $l$ be a non-negative integer. The following statements are equivalent:

(1) There doesn't exist an $l$ space-bounded accepting computation tree of M on $x$.

(2) There exists a regular $\mathrm{RG}(l)$ of $\mathrm{M}$ on $x$.

Lemma 4.1. Let

$\mathrm{L}_{5}=\left\{B(1) \# B(2) \# \ldots \# B(n) c w_{1} c w_{2} c \ldots c w_{k} c c w_{1}^{\prime} c w_{2}^{\prime} c \ldots c w_{r}^{\prime} \in\{0,1, \#, c\}^{+} \mid\right.$ $n \geq 2 \& k \geq 1 \& r \geq 1 \& \forall i(1 \leq i \leq k)\left[w_{i} \in\{0,1\}^{+}\right] \&$

$\forall j(1 \leq j \leq r-1)\left[w_{j}^{\prime} \in\{0,1\}^{+}\right] \& w_{r}^{\prime} \in\{0,1\}^{\lceil\log n\rceil} \&$

$\left.\forall l(1 \leq l \leq k)\left[w_{l} \neq w_{r}^{\prime}\right]\right\}$, where for each $m(1 \leq m \leq n), \mathrm{B}(m)$ denotes

the binary representation (with no leading zeros) of the integer $m$,

$\mathrm{L}_{6}=\left\{c w \mid w \in\{0,1\}^{+}\right\}^{*}$,

$\mathrm{L}_{7}=\left\{B(1) \# B(2) \# \ldots \# B(n) c w_{1} c w_{2} c \ldots c w_{k} c c w_{1}^{\prime} c w_{2}^{\prime} c \ldots c w_{r}^{\prime} \in\{0,1, \#, c\}^{+} \mid\right.$

$\left.n \geq 2 \& k \geq 1 \& r \geq 1 \& \forall i(1 \leq i \leq k) \forall j(1 \leq j \leq r)\left[w_{i}, w_{j}^{\prime} \in\{0,1\}^{+}\right]\right\}$, and

$\mathrm{L}_{8}=\left\{B(1) \# B(2) \# \ldots \# B(n) c w_{1} c w_{2} c \ldots c w_{k} c c_{1} w_{1}^{\prime} c_{2} w_{2}^{\prime} \ldots c_{r} w_{r}^{\prime} \in\{0,1, \#, c, d\}^{+} \mid\right.$

$n \geq 2 \& k \geq 1 \& r \geq 1 \& \exists i(1 \leq i \leq r)\left[c_{i}=d \& w_{i}^{\prime} \in\{0,1\}^{\lceil\log n\rceil} \&\right.$

$\left.\left.\forall j(1 \leq j \leq k)\left[w_{j} \neq w_{i}^{\prime}\right] \& \forall l(1 \leq l \leq r, l \neq i)\left[c_{l}=c \& w_{l}^{\prime} \in\{0,1\}^{+}\right]\right]\right\}$.

Then

(1) $\mathrm{L}_{5} \in$ strong-ASPACE $(\log \log n)$,

(2) $\mathrm{L}_{5} \cup \mathrm{L}_{6} \in$ strong-ASPACE $(\log \log n)$,

(3) $\mathrm{L}_{7} \in$ strong-ASPACE $(\log \log n)$,

(4) $\mathrm{L}_{8} \in$ strong-ASPACE $(\log \log n)$, and

(5) $\mathrm{L}_{5} \mathrm{~L}_{6} \notin$ weak-ASPACE $(o(\log n))$.

Proofs of (1)-(4): By standard techniques as in [10]. We leave the proofs to the reader as an easy exercise.

Proof of (5): Suppose to the contrary there is a weakly $\mathrm{L}(n)$ space-bounded $2 \mathrm{aTm} \mathrm{M}$ which accepts $\mathrm{L}_{5} \mathrm{~L}_{6}$.

For each $n \geq 2$, let

$\mathrm{W}(n)=\left\{\mathrm{B}(1) \# \mathrm{~B}(2) \# \ldots \# \mathrm{~B}(n) c w_{1} c w_{2} c \ldots c w_{p(n)} c c w_{1} c w_{2} c \ldots c w_{p(n)} \mid \forall i(1 \leq i \leq p(n))\right.$ $\left.\left[w_{i} \in\{0,1\}^{\lceil\log n\rceil}\right]\right\}$, where $p(n)=2^{\lceil\log n\rceil}$.

As easily seen, each $x$ in $\mathrm{W}(n)$ is not in $\mathrm{L}_{5} \mathrm{~L}_{6}$. Thus, from Fact 4.1 , for each $x \in \mathrm{W}(n)$, there exists a fixed regular reduced graph of $\mathrm{L}(r(n))$ space-bounded rejecting computation tree of $\mathrm{M}$ on $x$, where $r(n)$ is the length of each word in $\mathrm{W}(n)$ and $r(n)=O(n \log n)$. We denote this graph by $\mathrm{G}(x)$.

For each $x=\mathrm{B}(1) \# \mathrm{~B}(2) \# \ldots \# \mathrm{~B}(n) c w_{1} c w_{2} c \ldots c w_{p(n)} c c w_{1} c w_{2} c \ldots c w_{p(n)}$ in $\mathrm{W}(n)$, 
we call the left part of $x$ (i.e., $\left.\mathrm{B}(1) \# \mathrm{~B}(2) \# \ldots \# \mathrm{~B}(n) c w_{1} c w_{2} c \ldots c w_{p(n)}\right)$ the left segment of $x$, and the right part of $x$ (i.e., $\left.c c w_{1} c w_{2} c \ldots c w_{p(n)}\right)$ the right segment of $x$.

For each $x \in \mathrm{W}(n)$, we partition $\mathrm{V}(\mathrm{G}(x))$, the set of nodes of $\mathrm{G}(x)$, as follows:

$$
\mathrm{V}(\mathrm{G}(x))=\mathrm{V}_{\text {left }}(\mathrm{G}(x)) \cup \mathrm{V}_{\text {right }}(\mathrm{G}(x)),
$$

where $\mathrm{V}_{\text {left }}(\mathrm{G}(x))$ (resp., $\mathrm{V}_{\text {right }}(\mathrm{G}(x))$ ) denotes the set of nodes of $\mathrm{G}(x)$ which are labeled by configurations representing that the input head of $\mathrm{M}$ is on the left segment of $x$ or on the left endmaker $\not$ (resp., on the right segment of $x$ or on the right endmaker $\$$ ).

We then extract the set set of nodes in $\mathrm{V}_{\text {left }}\left(\mathrm{G}(x)\right.$ ) (resp., $\mathrm{V}_{\text {right }}(\mathrm{G}(x))$ ) that are labeled by configurations which $\mathrm{M}$ enters just after the input head crosses from the right segment of $x$ to the left segment of $x$ (resp., from the left segment of $x$ to the right segment of $x$ ). That is, we have

$$
\begin{aligned}
& \mathrm{V}_{\text {left }}^{\leftarrow}(\mathrm{G}(x))=\left\{v \in \mathrm{V}_{\text {left }}(\mathrm{G}(x)) \mid\left(v^{\prime}, v\right) \in \mathrm{E}(\mathrm{G}(x)) \& v^{\prime} \in \mathrm{V}_{\text {right }}(\mathrm{G}(x))\right\}, \\
& \mathrm{V}_{\text {right }}^{\vec{f}}(\mathrm{G}(x))=\left\{v \in \mathrm{V}_{\text {right }}(\mathrm{G}(x)) \mid\left(v^{\prime}, v\right) \in \mathrm{E}(\mathrm{G}(x)) \& v^{\prime} \in \mathrm{V}_{\text {left }}(\mathrm{G}(x))\right\},
\end{aligned}
$$

where $\mathrm{E}(\mathrm{G}(x))$ denotes the set of edges of $\mathrm{G}(x)$.

Furthermore, we partition $\mathrm{E}(\mathrm{G}(x))$ as follows: where

$\mathrm{E}(\mathrm{G}(x))=\mathrm{E}_{\text {left }}(\mathrm{G}(x)) \cup \mathrm{E}_{\text {right }}(\mathrm{G}(x)) \cup \mathrm{E}_{\leftarrow}(\mathrm{G}(x)) \cup \mathrm{E}_{\rightarrow}(\mathrm{G}(x))$,

$\mathrm{E}_{\text {left }}(\mathrm{G}(x))=\left\{\left(v, v^{\prime}\right) \in \mathrm{E}(\mathrm{G}(x)) \mid v \in \mathrm{V}_{\text {left }}(\mathrm{G}(x)) \& v^{\prime} \in \mathrm{V}_{\text {left }}(\mathrm{G}(x))\right\}$,

$\mathrm{E}_{\text {right }}(\mathrm{G}(x))=\left\{\left(v, v^{\prime}\right) \in \mathrm{E}(\mathrm{G}(x)) \mid v \in \mathrm{V}_{\text {right }}(\mathrm{G}(x)) \& v^{\prime} \in \mathrm{V}_{\text {right }}(\mathrm{G}(x))\right\}$,

$\mathrm{E}_{\leftarrow}(\mathrm{G}(x))=\left\{\left(v, v^{\prime}\right) \in \mathrm{E}(\mathrm{G}(x)) \mid v \in \mathrm{V}_{\text {right }}(\mathrm{G}(x)) \& v^{\prime} \in \mathrm{V}_{\text {left }}^{\leftarrow}(\mathrm{G}(x))\right\}$, We let$$
\mathrm{E}_{\rightarrow}(\mathrm{G}(x))=\left\{\left(v, v^{\prime}\right) \in \mathrm{E}(\mathrm{G}(x)) \mid v \in \mathrm{V}_{\text {left }}(\mathrm{G}(x)) \& v^{\prime} \in \mathrm{V}_{\text {right }}^{\rightarrow}(\mathrm{G}(x))\right\} .
$$

Cross-Pair $(\mathrm{G}(x))=<\operatorname{label}\left(\mathrm{V}_{\text {left }}^{\leftarrow}(\mathrm{G}(x))\right), \operatorname{label}\left(\mathrm{V}_{\text {right }}^{\rightarrow}(\mathrm{G}(x))\right)>$.

For each word $x=\mathrm{B}(1) \# \mathrm{~B}(2) \# \ldots \# \mathrm{~B}(n) c w_{1} c w_{2} c \ldots c w_{p(n)} c c w_{1} c w_{2} c \ldots c w_{p(n)} \in$ $\mathrm{W}(n)$, let $\operatorname{contents}(x)=\left\{w \in\{0,1\}^{\lceil\log n\rceil} \mid w=w_{i}\right.$ for some $\left.1 \leq i \leq p(n)\right\}$. For any two words $x, y \in \mathrm{W}(n)$, divide $\mathrm{W}(n)$ into contents-equivalence classes by making $x$ and $y$ contents-equivalent if $\operatorname{contents}(x)=\operatorname{contents}(y)$.

There are

$$
\operatorname{contents}(n)=\left(\begin{array}{c}
p(n) \\
1
\end{array}\right)+\left(\begin{array}{c}
p(n) \\
2
\end{array}\right)+\ldots+\left(\begin{array}{c}
p(n) \\
p(n)
\end{array}\right)=2^{p(n)}-1
$$

contents-equivalence classes.

We denote by CONTENTS $(n)$ the set of all the representatives arbitrarily chosen from these contents $(n)$ contents-equivalence classes. Of course,

$$
|\operatorname{CONTENTS}(n)|=\operatorname{contents}(n)=2^{p(n)}-1 .
$$

Proposition 4.1 For two different elements $x, y \in \operatorname{CONTENT}(n)$,

$$
\text { Cross-Pair }(\mathrm{G}(x)) \neq \text { Cross-Pair }(\mathrm{G}(y)) \text {. }
$$

[Proof. Suppose to the contrary that Cross-Pair $(\mathrm{G}(x))=$ Cross-Pair $(\mathrm{G}(y))$. From $\mathrm{G}(x)$ and $\mathrm{G}(y)$, we construct the following graph $\mathrm{G}(x) \emptyset \mathrm{G}(y)$ :

$$
\begin{aligned}
& \mathrm{V}(\mathrm{G}(x) \emptyset \mathrm{G}(y))=\mathrm{V}_{\text {left }}(\mathrm{G}(x)) \cup \mathrm{V}_{\text {right }}(\mathrm{G}(y)), \\
& \mathrm{E}(\mathrm{G}(x) \oplus \mathrm{G}(y))=\mathrm{E}_{\text {left }}(\mathrm{G}(x)) \cup \mathrm{E}_{\text {right }}(\mathrm{G}(y)) \cup \mathrm{E}_{\leftarrow} \cup \mathrm{E}_{\rightarrow},
\end{aligned}
$$
where 
$\mathrm{E}_{\leftarrow}=\left\{\left(u, v^{\prime}\right) \in \mathrm{V}_{\text {right }} \mathrm{G}(y)\right) \times \mathrm{V}_{\text {left }}^{\leftarrow}(\mathrm{G}(x)) \mid\left(u, u^{\prime}\right) \in \mathrm{E}_{\leftarrow}(\mathrm{G}(y)) \&\left(v, v^{\prime}\right) \in \mathrm{E}_{\leftarrow}(\mathrm{G}(x)) \&$ $\left.\operatorname{label}\left(u^{\prime}\right)=\operatorname{label}\left(v^{\prime}\right)\right\}$, and

$\mathrm{E}_{\rightarrow}=\left\{\left(v, u^{\prime}\right) \in \mathrm{V}_{\text {left }} \mathrm{G}(x)\right) \times \mathrm{V}_{\text {right }}^{\rightarrow}(\mathrm{G}(y)) \mid\left(v, v^{\prime}\right) \in \mathrm{E}_{\rightarrow}(\mathrm{G}(x)) \&\left(u, u^{\prime}\right) \in \mathrm{E}_{\rightarrow}(\mathrm{G}(y)) \&$ $\left.\operatorname{label}\left(u^{\prime}\right)=\operatorname{label}\left(v^{\prime}\right)\right\}$.

Intuitively, $\mathrm{G}(x) \oplus \mathrm{G}(y)$ is the graph obtained by connecting the part of $\mathrm{G}(x)$ which correspondes to the left segment of $x$ with the part of $\mathrm{G}(y)$ which correspondes to the right segment of $y$ (see Fig.1). From our assumption that Cross-Pair $(\mathrm{G}(x))=$ Cross-Pair $(\mathrm{G}(y))$, it is easy to see that the following fact holds.

Fact 4.2. (1) For any $v \in \mathrm{V}_{\text {left }}(\mathrm{G}(x))$, label $\left(\delta_{\mathrm{G}(x)}^{+} \emptyset_{\mathrm{G}(y)}(v)\right)=\operatorname{label}\left(\delta_{\mathrm{G}(x)}^{+}(v)\right)$, and

(2) for any $v \in \mathrm{V}_{\text {right }}(\mathrm{G}(y))$, label $\left(\delta_{\mathrm{G}(x)}^{+} \bigoplus_{\mathrm{G}(y)}(v)\right)=\operatorname{label}\left(\delta_{\mathrm{G}(y)}^{+}(v)\right)$.

We assume without loss of generality that

$$
\operatorname{contents}(y)-\operatorname{contents}(x) \neq \emptyset \text { (empty set). }
$$

Now, consider the word $z_{1} z_{2}$ such that

(i) $z_{1}$ is identical with the left segment of $x$, and

(ii) $z_{2}$ is identical with the right segment of $y$.

Let $v_{0}$ be the node of $\mathrm{G}(x)$ labeled by $\mathrm{I}_{M}$ (note that $v_{0}$ is in $\mathrm{V}(\mathrm{G}(x) \oplus \mathrm{G}(y)$ ). We consider the following depth-first search on $\mathrm{V}(\mathrm{G}(x) \emptyset \mathrm{G}(y))$ starting at $v_{0}$ : (1) $v:=v_{0}$

(2) for each $v_{i} \in \mathrm{V}(\mathrm{G}(x) \emptyset \mathrm{G}(y))$ such that $\delta^{+}(v)=\left\{v_{1}, v_{2}, \ldots, v_{k}\right\}$ :

- if $v_{i}$ has not been searched, then set $v:=v_{i}$ and repeat (2).

- if every $v_{i}$ in $\delta^{+}(v)$ has searched, then return to $v$.

From Fact 4.2 we can easily see that the sequence of values of variable $v$ above constructs a regular reduced graph of $\mathrm{L}(r(n))$ space-bounded rejecting computation tree of $\mathrm{M}$ on $z_{1} z_{2}$. This contradicts the fact that $z_{1} z_{2}$ is in $\mathrm{L}_{5} \mathrm{~L}_{6}$. This completes the proof of Proposition 4.1.]

For each $n \geq 2$,

$$
\mathrm{C}(n)=\{\text { Cross-Pair }(\mathrm{G}(x)) \mid x \in \operatorname{CONTENTS}(n)\} .
$$

Then

$$
|\mathrm{C}(n)| \leq 2^{2 \cdot e[n]}
$$

where $e[n]=s \mathrm{~L}(r(n)) t^{\mathrm{L}(r(n))}, s$ and $t$ are the numbers of states and work tape symbols of $\mathrm{M}$, respectively.

Since $\mathrm{L}(n)=o(\log n)$, it follows that for large $n$,

$$
\text { contents }(n)>\mathrm{C}(n) \text {. }
$$

Therefore, such a large $n$, there must exist two different $x, y$ in $\operatorname{CONTENTS}(n)$ such that Cross-Pair $(\mathrm{G}(x))=$ Cross-Pair $(\mathrm{G}(y))$. This contradicts Proposition 4.1, which completes the proof of Lemma 4.1 (5).

By using Lemma 4.1., we can get the following theorem.

Theorem 4.1. For each function $\log \log n \leq \mathrm{S}(n)=o(\log n)$, weak-ASPACE $(\mathrm{S}(n))$ and strong-ASPACE $(\mathrm{S}(n))$ are not closed under concatenation, Kleene closure, 
and length-preserving homomorphism.

Proof. Let $\mathrm{L}_{i}, i \in\{5,6,7,8\}$, be the languages described in Lemma 4.1.

Concatenation: Nonclosure under concatenation follows from Lemma 4.1 (1) and (5), and from the obvious fact that $\mathrm{L}_{5} \in$ strong-ASPACE$(\log \log \mathrm{n})$.

Kleene closure: It follows that $\left(\mathrm{L}_{5} \cup \mathrm{L}_{6}\right)^{*} \cap \mathrm{L}_{7}=\mathrm{L}_{5} \mathrm{~L}_{6} \notin$ weak-ASPACE$(\mathrm{o}(\log \mathrm{n}))$ (from Lemma 4.1 (5)). From this, Lemma 4.1 (2) and (3), and from the obvious fact that strong-ASPACE $(\mathrm{L}(n))$ and weak-ASPACE $(\mathrm{L}(n))$ are closed under intersection for any function $\mathrm{L}(n)$, nonclosure under Kleene closure follows.

Length-preserving homomorphism: Nonclosure under length-preserving homomorphism follows from Lemma 4.1 (4) and (5), and from the fact that $h\left(\mathrm{~L}_{8}\right)=\mathrm{L}_{5} \mathrm{~L}_{6}$, where $h:\{0,1, \#, c, d\} \rightarrow\{0,1, \#, c\}$ is a length-preserving homomorphism such that $h(0)=0, h(1)=1, h(\#)=\#$ and $h(c)=h(d)=c$.

\section{Conclusion}

We conclude this paper by giving the following open problem:

- Is closed PSPACE $(\mathrm{L}(n))$ under concatenation, Kleene closure and lengthpreserving homomorphism for $\log \log n \leq \mathrm{L}(n)=o(\log n)$ ? 


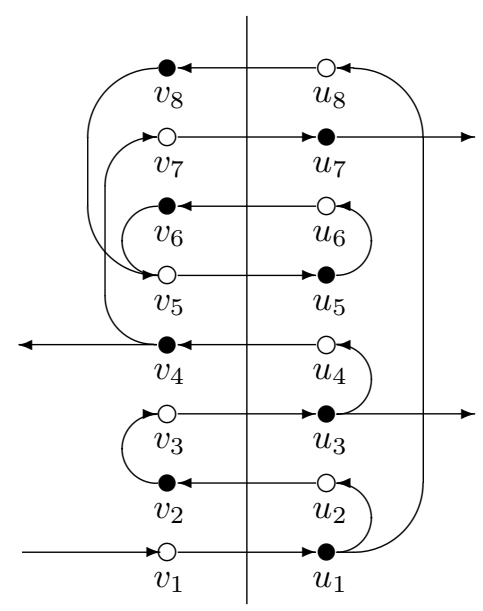

(1) $\mathrm{G}(x)$

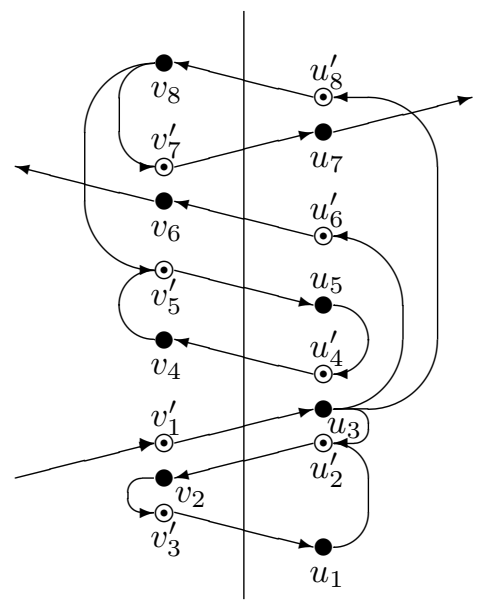

(2) $\mathrm{G}(y)$

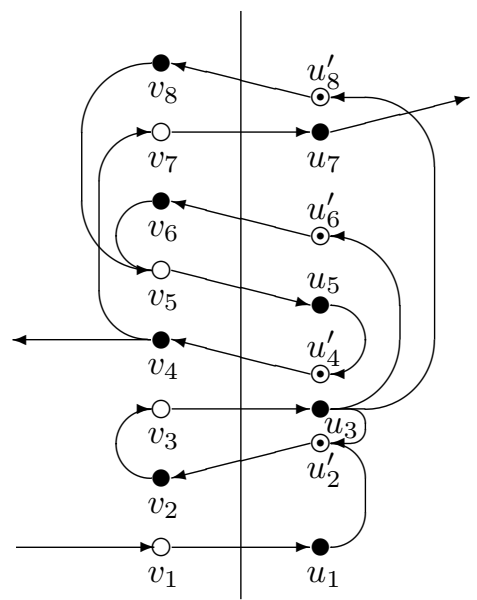

(3) $\mathrm{G}(x) \oplus \mathrm{G}(y)$

Fig. 1. Connection of graphs $\mathrm{G}(x)$ and $\mathrm{G}(y)$, where for simplicity, we identify node $v$ with its lavel, $\operatorname{label}(v)$. 


\section{References}

[1] B. V. Braunmühl, R. Gengler and R. Rettinger, "The alternation hierarchy for subalgorithmic space is infinite", Comput. Complexity 3, pp. 207-230, 1993.

[2] J. H. Chang, O. H. Ibarra and B. Ravikumar, "Some observations concerning alternating Turing machines using small space", Information Processing Letters 25, pp. 1-9, 1987.

[3] C. Dwork and L. Stockmeyer, "A time complexity gap for two-way probabilistic finite-state automata", SIAM J. COMPUT. 19, pp. 1011-1023, 1990.

[4] R. Freivalds, "Probabilistic two-way machines", Proceedings of the International Symposium on Mathematical Foundations of Computer Science, LNCS 118, pp. 33-45, 1981.

[5] R. Freivalds and M. Karpinski, "Lower space bounds for randomized computation", Proceedings of ICALP'94, LNCS 820, pp. 580-592, 1994.

[6] V. Geffert, "A hierarchy that does not collapse: alternations in low level space", Informatique théorique et Applications / Theoretical Informatics and Applications 28, no.5, pp. 462-512, 1994.

[7] J. Gill, "Computational complexity of probabilistic Turing machines", SIAM J. COMPUT. 6, no.4, pp. 675-695, 1977.

[8] A.G. Greenberg and A. Weiss, "A lower bound for probabilistic algorithms for finite state machines", JCSS, Vol. 33, pp. 88-105, 1986.

[9] J. E. Hopcroft and J. D. Ullman, "Formal Languages and Their Relation to Automata", Addison-Wesley, Reading, Mass., 1969.

[10] A. Ito, K. Inoue and I. Takanami, "A note on alternating Turing machines using small space", IEICE Trans. E 70 (10), pp. 990-996, 1987.

[11] A. Ito, T. Okazaki, K. Inoue and Y. Wang, "Nonclosure under complementation of two-dimensional alternating $o(\log \log m)$ space complexity class", IEICE Trans. Vol. I81-D-I, no 6, pp. 593-603, 1998.

[12] K. Iwama, "ASPACE $(o(\log \log n))$ is regular", SIAM J. COMPUT. 22, pp. 207-221, 1993.

[13] M. Liśkiewicz and R. Reischuk, "The sublogarithmic alternating space world", SIAM J. COMPUT. 25, pp. 828-861, 1996.

[14] J. Wang, " A note on two-way probabilistic automata", Information Processing Letters 43, pp. 321-326, 1992. 